\title{
Exenatide regulates inflammation and the production of reactive oxygen species via inhibition of S1PR2 synthesis
}

\author{
Yunyun Yang ${ }^{A-E}$, Qingyi Zhao ${ }^{A-C, E, F}$ \\ Department of Ophthalmology, Zhangjiagang TCM Hospital (affiliated to Nanjing University of Chinese Medicine), China \\ A - research concept and design; $B$ - collection and/or assembly of data; $C$ - data analysis and interpretation; \\ $D$ - writing the article; $E$ - critical revision of the article; $F$ - final approval of the article
}

\section{Address for correspondence}

Yunyun Yang

E-mail:zhaoqingyi77@126.com

Funding sources

None declared

Conflict of interest

None declared

\section{Acknowledgements}

We wish to acknowledge Dr. Qinghuai Liu, Professor of Jiangsu Provincial People's Hospital, Nanjing Medical University, China, for his help in interpreting the significance of this study results. We would like to acknowledge the authors on the list for their respective contributions to the paper.

Received on July 13,2020 Reviewed on July 24, 2020 Accepted on February 19, 2021

Published online on May 13, 2021

\begin{abstract}
Background. Microvascular dysfunction is one of the most serious complications of diabetic retinopathy (DR). As a novel treatment drug for type 2 diabetes, exenatide possesses protective properties against retinal neurodegeneration. Sphingosine-1-phosphate receptor 2 (S1PR2) could regulate blood glucose in diabetes, and inhibition of STPR2 is involved in the treatment of diabetes. However, the mechanism of exenatide in human retinal vascular endothelial cells (hRVECs) has not been fully defined.

Objectives. We tested the hypothesis that S1PR2 plays a vital role in high glucose (HG)-induced hRVECS, and that exenatide could ameliorate HG-induced hRVEC injury by regulating SIPR2 production.

Materials and methods. The hRVECs underwent HG-stimulation. Quantitative real-time polymerase chain reaction (RT-qPCR) and western blot were performed to examine the expression of S1PR2. Oxidative stress levels, inflammatory markers and cell apoptosis were detected using reactive oxygen species (ROS) staining, enzyme-linked immunosorbent assay (ELLSA) kits and TUNEL staining.

Results. High glucose increased the level of S1PR2 in hRVECs and reduced the expression of glucagon-like peptide-1 receptor (GLP1R) compared to the control group. Exenatide decreased the level of S1PR2 induced by HG. Sphingosine-1 blocked the effects of exenatide, alleviating the ROS and cell apoptosis induced by HG. JTE-013 treatment protected hRVECs from injury by HG. The inhibitory effects of exenatide on S1PR2 expression lessened HG-induced hRVEC injury.
\end{abstract}

Conclusions. The results demonstrate a possible mechanism of exenatide mediated inhibition of S1PR2 synthesis, and support S1PR2 as a novel target for treating DR.

Key words: diabetic retinopathy, high glucose, exenatide, SIPR2

Cite as

Yang Y, Zhao Q. Exenatide regulates inflammation and the production of reactive oxygen species via inhibition of S1PR2 synthesis. Adv Clin Exp Med. 2021;30(5):555-561. doi:10.17219/acem/133483

DOI

10.17219/acem/133483

Copyright

Copyright by Author(s)

This is an article distributed under the terms of the

Creative Commons Attribution 3.0 Unported (CC BY 3.0)

(https://creativecommons.org/licenses/by/3.0/) 


\section{Background}

Diabetic retinopathy (DR) is a common complication of diabetes mellitus, which can lead to blindness in severe cases. The primary source of damage to retinal microvessels is peroxide damage caused by high glucose (HG). ${ }^{1}$ Exenatide, a novel type 2 diabetes treatment drug, can simulate the activity of endogenous glucagon-like peptide-1 (GLP-1) while resisting degradation by dipeptidyl peptidase IV. Studies have shown that exenatide shows similar effects to GLP-1. For example, exenatide stimulates insulin secretion in a glucose-dependent manner, reduces plasma glucagon levels, inhibits gastric emptying and food intake, reduces body weight, and delays $\beta$-cell dysfunction to a certain extent. ${ }^{2}$

Regulators of GLP-1/GLP-1 receptor (GLP-1R) signaling could also have parallel roles in $\mathrm{S} 1 \mathrm{P} /$ sphingosine-1-phosphate receptor 2 (S1P/S1PR2) signaling. ${ }^{3,4}$ Human retinal S1PR2 is reported to be expressed in vascular endothelial cells. ${ }^{5}$ In a high-sugar environment, increased S1P activates S1PR2, and, in turn, the intracellular signaling pathway, which plays important roles in cell proliferation, inflammation regulation and angiogenesis. ${ }^{6}$ The S1PR2 antagonist JTE-013 can prevent cell damage and inhibit the elevation of blood glucose in diabetic mice due to its glucose reducing activity. ${ }^{7,8}$ Sphingosine-1 may also regulate Akt-eNOS signaling in lung endothelial cells and affect vascular barrier functions in vivo. ${ }^{5}$ Therefore, the targeted inhibition of S1PR2 is important for the treatment of diabetes.

Inflammation has been reported to be a cause of diabetic retinal damage in the early stages of DR. ${ }^{9}$ Therefore, we also analyzed the effect of exenatide on inflammation. Reactive oxygen species (ROS) are greatly increased in the body during inflammation, which can lead to direct damage to lipid proteins and chromosomal nucleic acids, triggering cell apoptosis and the occurrence and development of the disease. Hyperglycemia causes the overproduction of ROS through various mechanisms, along with the imbalance of normal physiological functions of cells. ${ }^{10,11}$ There appears to be a vicious positive feedback cycle involving ROS, inflammation and cell apoptosis. ${ }^{12}$ However, whether the long-lasting ligand GLP-1R is also involved in retinal vascular endothelial cell injury induced by HG through targeted inhibition of S1PR2 still needs to be confirmed. Preliminary experiments have shown that exenatide decreases S1PR2 levels in retinal vascular endothelial cells when induced by HG.

\section{Objectives}

This study explores whether exenatide reduces retinal vascular endothelial cell injury by targeting S1PR2 expression.

\section{Materials and methods}

\section{Cell line}

Human retinal vascular endothelial cells (hRVECs) were purchased from Shanghai Cell Bank, Chinese Academy of Sciences, Shanghai, China. They are adherent cells that can be passed on at a confluence rate of $80 \sim 90 \%$ in lowglucose Dulbecco's modified Eagle's medium (L-DMEM; Gibco, Waltham, USA) containing $10 \%$ fetal bovine serum (FBS), $100 \mathrm{U} / \mathrm{mL}$ of penicillin and $100 \mathrm{U} / \mathrm{mL}$ of streptomycin (Sigma-Aldrich, St. Louis, USA). The hRVECs at logarithmic growth stage were digested with pancreatin, and resuspended to a concentration of $3 \times 10^{4}$ cells/mL in L-DMEM medium containing 1\% FBS. The cells were then divided into a control group (normal saline), mannitol group (MG, $25 \mathrm{mmol} / \mathrm{L}$ ), HG group (HG, $25 \mathrm{mmol} / \mathrm{L}$ ), MG+exenatide group, $\mathrm{HG}+$ exenatide group, HG+exenatide+S1P $(1 \mu \mathrm{m})$ group, and HG+JTE-013 $(0.1 \mu \mathrm{m})$ group. Exenatide, S1P or JTE-013 were added to the cells as soon as the hyperglycemia cell model was established. After treatment for $48 \mathrm{~h}$, further experiments were performed.

\section{RT-qPCR}

After $48 \mathrm{~h}$ of treatment, the total RNA of each group was extracted with Trizol reagent (Trizol, Waltham, USA), and reverse-transcribed into cDNA (TaKaRa, Tokyo, Japan). The synthesized cDNA was used as a template for quantitative experiments on an ABI 7100 quantitative fluorescence polymerase chain reaction (PCR) instrument (Applied Biosystems, Waltham, USA). The reaction system for RNA amplification was prepared as follows: RNase-free deionized water $(10 \mu \mathrm{L})$, cDNA $(1 \mu \mathrm{L})$, upstream and downstream primers $(0.5 \mu \mathrm{L}$, respectively), and SYBR Premix $(8 \mu \mathrm{L})$. GAPDH mRNA was used as an internal reference. The relative levels of S1PR1, S1PR2, S1PR3, and S1PR4 mRNA were calculated using the $2^{-\Delta \Delta \mathrm{Ct}}$ method.

\section{ELISA}

The hRVECs were seeded into six-well plates. Cells were cultured as indicated above. After transfection for $48 \mathrm{~h}$, cells were collected to detect the contents of tumor necrosis factor $\alpha$ (TNF- $\alpha$ ), interleukin (IL)- $1 \beta$ and IL- 6 protein in each group, using TNF- $\alpha$, IL- $1 \beta$ and IL- 6 enzyme-linked immunosorbent assay (ELISA) kits provided by Thermo Fisher Scientific (Waltham, USA).

\section{ROS staining}

Intracellular ROS were detected using dichloro-dihydrofluorescein (DCFH) fluorescent probe (Nanjing Jiancheng Bioengineering Institute, Nanjing, China). The hRVECs were seeded into six-well plates and $20 \mu \mathrm{mol}$ of DCFH 
fluorescence probes were added to all cells except for the negative control group. The samples were incubated in darkness for $45 \mathrm{~min}$, washed with phosphate-buffered saline (PBS) 3 times, then observed under an inverted fluorescence microscope (Nikon Eclipse Ti; Nikon Corp., Tokyo, Japan) and photographed. The fluorescence intensity was detected through a fluorescence microplate (Bio-Rad, Hercules, USA). The utilized excitation wavelength was $488 \mathrm{~nm}$ and the emission wavelength was $525 \mathrm{~nm}$.

\section{TUNEL staining}

Apoptotic cells were stained according to the instructions for the TUNEL kit (Beyotime, Shanghai, China). Cells were washed with PBS and then fixed with $4 \%$ paraformaldehyde. Phosphate-buffered saline with $0.1 \%$ Triton $\mathrm{X}-100$ was added for incubation in an ice bath for 2 minutes. The TUNEL solution was prepared and added to cell sections. The tablets were sealed with anti-fluorescence quenching solution and observed under a fluorescence microscope (Olympus Corp., Tokyo, Japan).

\section{Western blot}

Total hRVEC protein was extracted, and SDS polyacrylamide gel was prepared after routine quantitative and denaturation. Routine sampling, electrophoresis, membrane transfer, and sealing were performed. The primary antibodies and GAPDH primary antibody (Abcam, Cambridge, UK) were added and incubated at $4^{\circ} \mathrm{C}$ overnight. Tris-buffered saline with Tween (TBST) was used to wash the membrane 3 times; then, the membrane was incubated with secondary antibody for $1 \mathrm{~h}$. The membrane was washed using TBST 3 times. Electrochemiluminescence (ECL) solution was added for $20 \mathrm{~s}$ in a dark room. The grey value was analyzed using ImageJ software (National Institutes of Health, Bethesda, USA).

\section{Statistical analyses}

GraphPad Prism v. 6.0 (GraphPad Software, San Diego, USA) was used for statistical analyses of the data. Comparison among multiple groups of samples was performed using one-way analysis of variance (ANOVA), followed by Tukey's test. All experiments were repeated at least 3 times. A value of $\mathrm{p}<0.05$ was considered as statistically significant.

\section{Results}

\section{Exenatide regulates the expression of GLP-1R and S1PR2 in hRVECs}

High glucose stimulated S1PR2 levels in hRVECs (Fig. 1A). In contrast to the control group, S1PR1, S1PR3 and S1PR4 levels showed only slight increases after HG induction (Fig. 1A). Mannitol had no effect on levels of S1PR1, S1PR2, S1PR3, or S1PR4.

We examined whether exenatide affected the production of S1PR2 protein. The levels of S1PR2 protein and S1PR2 mRNA in hRVECs stimulated by HG were both substantially higher than in the control group (Fig. 1B). Mannitol was used as an osmotic pressure control. In the MG group, exenatide did not affect either S1PR2 protein or S1PR2 mRNA levels. However, exenatide markedly reduced S1PR2 protein and S1PR2 mRNA levels in hRVECs stimulated by $\mathrm{HG}$, relative to the $\mathrm{HG}$ group (Fig. 1C). Exenatide, as an agonist of GLP-1R, exerted positive effects on GLP$1 \mathrm{R}$ expression in hRVECs. These findings suggest that exenatide reduced S1PR2 production in HG-stimulated hRVECs.

\section{Exenatide decreased inflammatory marker levels through S1PR2 regulation}

Inflammatory cytokines, such as IL- $1 \beta$ and TNF- $\alpha$, are highly expressed in the retinas of DR patients, leading to an inflammatory response and damage to the bloodretinal barrier, and thereby promoting the development of DR. We analyzed pro-inflammatory markers to assess inflammatory response induced HG in hRVECs. In contrast to the control group, HG markedly increased TNF- $\alpha$, IL-1 $\beta$ and IL-6 expression (Fig. 2). Significantly, inhibitory effects on these pro-inflammatory factors were observed when hRVECs were treated with both exenatide and HG (Fig. 2), which indicated a possible protective effect of exenatide against the inflammatory response. In addition, S1P, an agonist of S1PR2, counteracted the inhibition of inflammation mediators by exenatide. We also found that hRVECs in the HG+JTE-013 group exhibited similar responses to those in the HG+exenatide group. These data implied that exenatide could decrease the inflammatory response induced by HG through regulation of S1PR2 protein activity.

\section{Exenatide reduces ROS levels through S1PR2}

Disruption of the balance between the formation and clearance rates of free radicals can produce oxidative stress and cell damage. Therefore, we analyzed the effects of exenatide on ROS and explored the regulation mechanism, using either S1P or JTE-013, an effective and selective S1PR2 antagonist. Exenatide reduced ROS levels, as revealed through ROS staining and the detection of fluorescence intensity (Fig. 3), whereas S1P counteracted exenatide-mediated ROS downregulation in HG-stimulated hRVECs. When JTE-013 was utilized to block the functions of S1PR2, ROS levels were significant decreased in HGstimulated hRVECs relative to the HG group. The results were consistent with the reported effects of exenatide. These observations implied that exenatide decreased ROS levels through downregulation of S1PR2 production. 

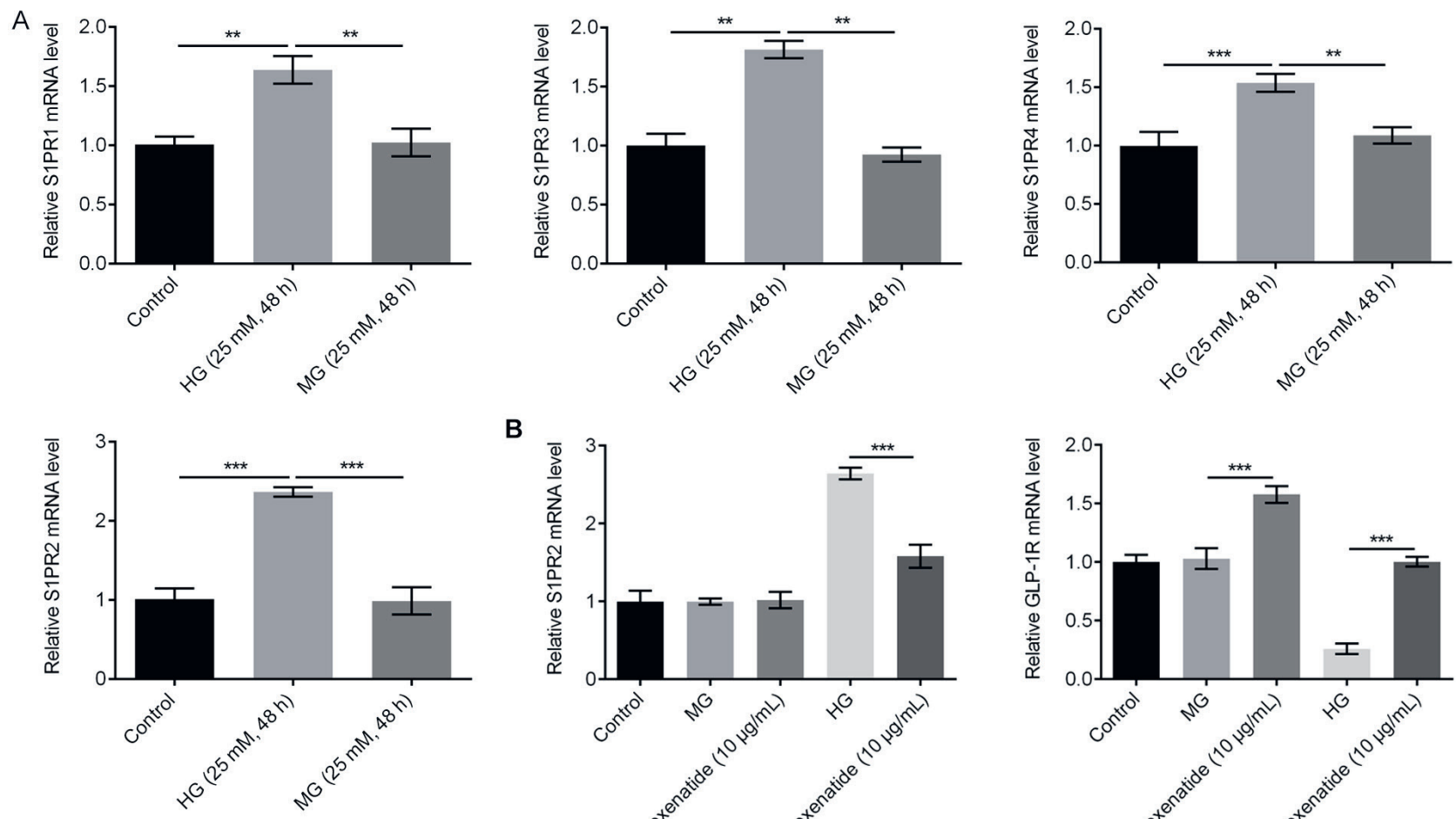

B
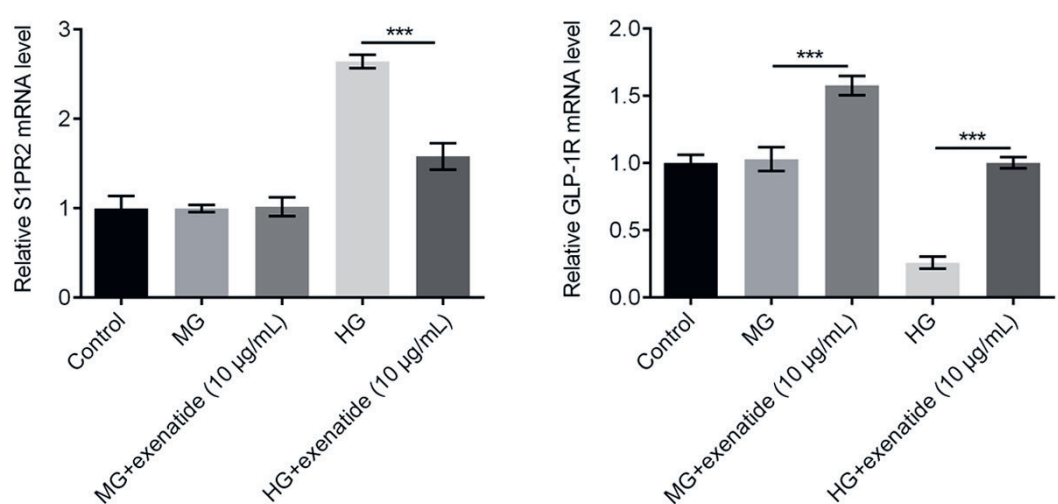

C
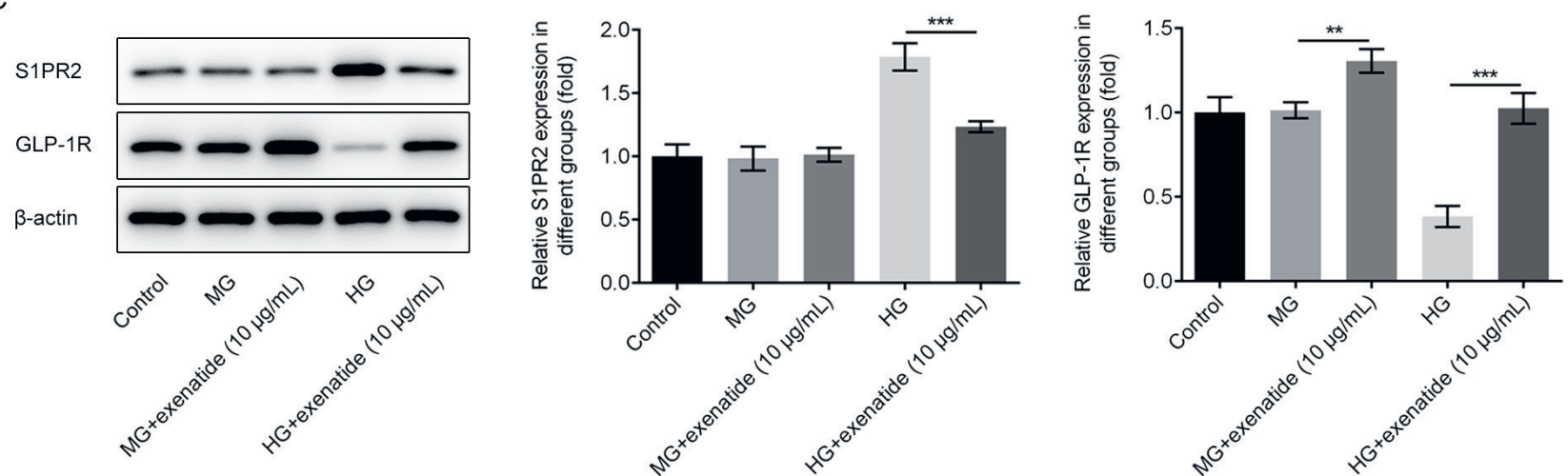

Fig. 1. A. Treatment with HG (25 mM) for $48 \mathrm{~h}$ stimulated S1PR2 production in hRVECs, as seen with RT-qPCR and western blot; B and C. Exenatide $(10 \mathrm{\mu g} / \mathrm{mL})$ stimulated a decrease in S1PR2 production in hRVECs with HG. All results are presented as mean \pm SD percentage of the control level, based on 3 independent tests

${ }^{* *} p<0.01 ; * * * 0.001$
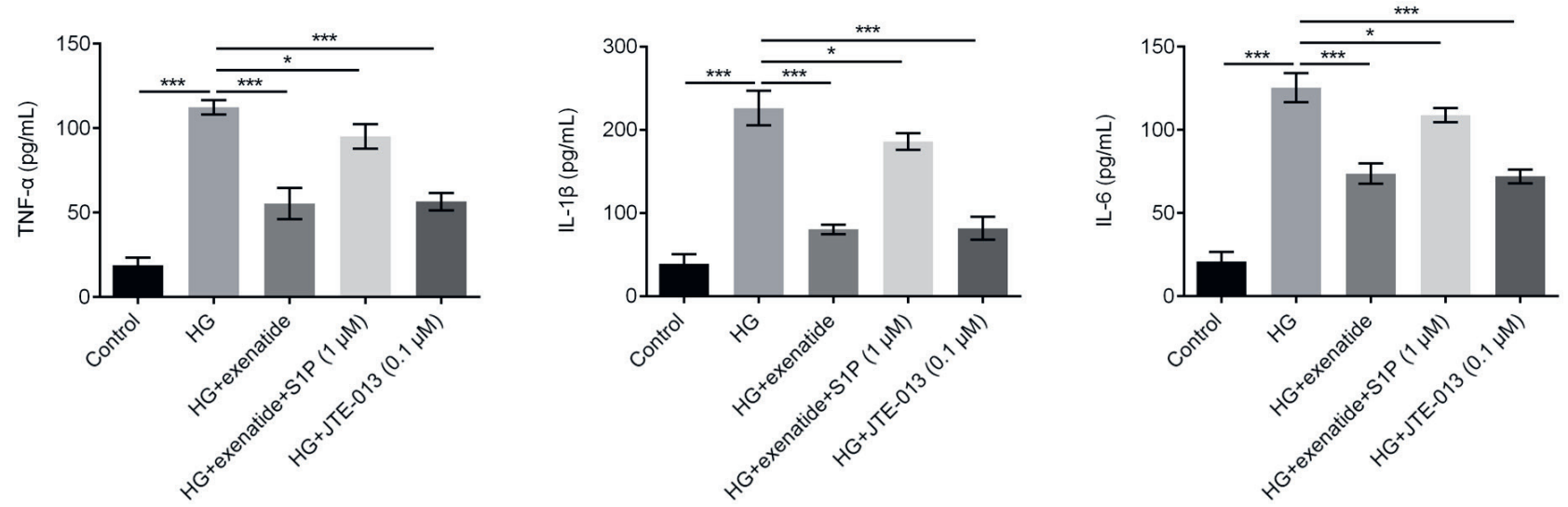

Fig. 2. Exenatide $(10 \mu \mathrm{g} / \mathrm{mL})$ reduced TNF-a, IL-1 $\beta$ and IL-6 expression through regulation of S1PR2. The results are expressed as mean \pm SD of the mean ${ }^{*} p<0.05 ;{ }^{* * *} p<0.001$. 

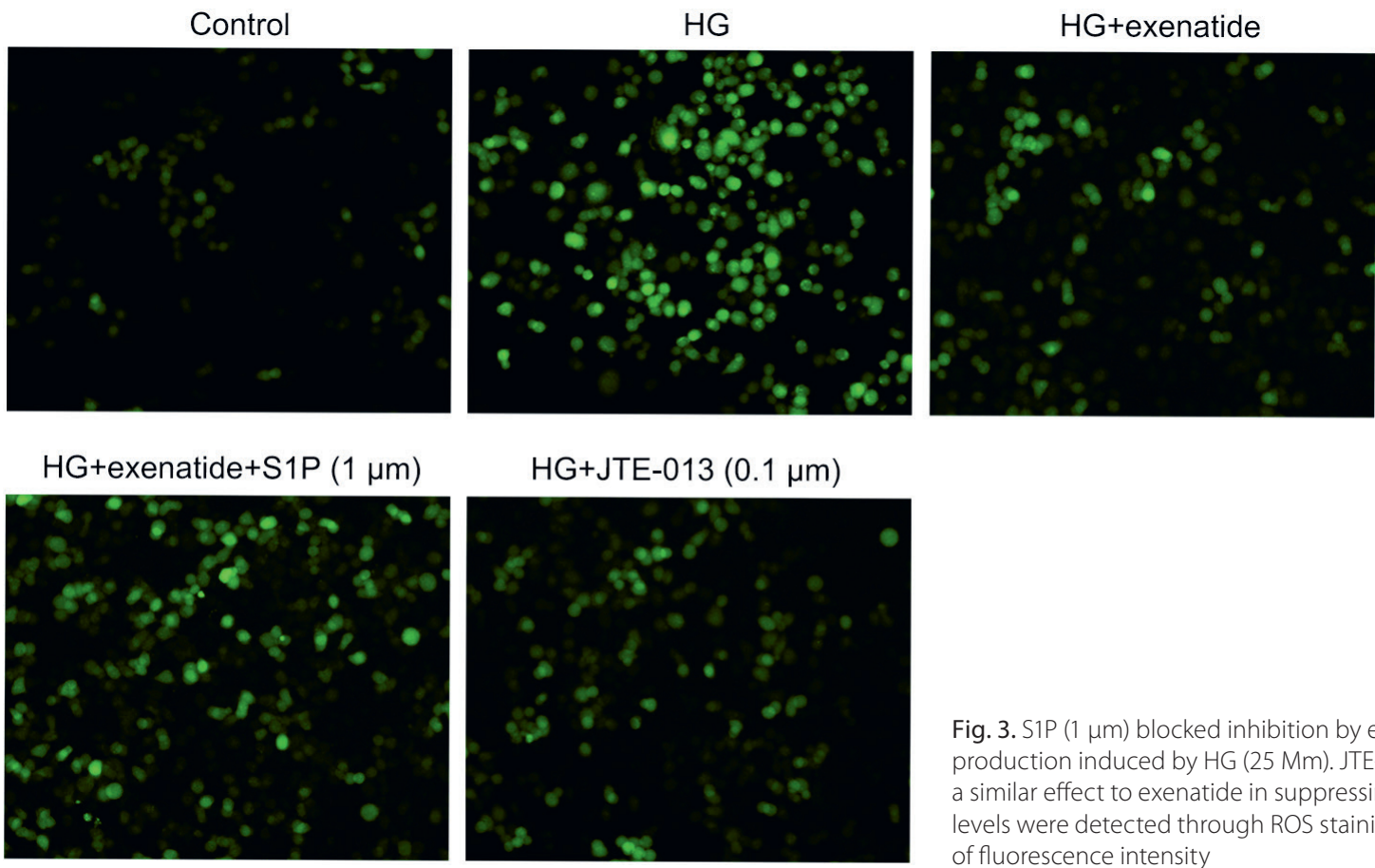

Fig. 3. S1P $(1 \mu \mathrm{m})$ blocked inhibition by exenatide of ROS production induced by HG $(25 \mathrm{Mm})$. JTE-013 $(0.1 \mu \mathrm{m})$ presented a similar effect to exenatide in suppressing ROS. The ROS levels were detected through ROS staining and the analysis of fluorescence intensity

\section{Exenatide lessened cell apoptosis triggered by HG through S1PR2}

TUNEL staining was performed to assess cell apoptosis levels, which revealed a dramatic increase of cell apoptosis in hRVECs exposed to HG (Fig. 4A,B). Simultaneously, exenatide treatment reduced HG-induced cell apoptosis, which could be blocked by addition of S1P. The S1PR2 antagonist markedly decreased apoptosis in hRVECs compared with the HG group. These results indicated that exenatide protected hRVECs from apoptosis induced by HG through S1PR2. Next, we explored how exenatide regulated cell apoptosis caused by HG. The expression of a panel of established apoptosis-related proteins was analyzed using western blot assay. As shown in Fig. $4 \mathrm{C}$, exenatide induced an increase of $\mathrm{Bcl} 2$, as well as a reduction of Bax, cleaved caspase- 3 and cleaved caspase-9.

\section{Discussion}

The present study provides evidence that exenatide protects cells against ROS injury, inflammatory damage and apoptosis, based on composite results obtained in HGinduced hRVECs. Our research also reveals the mechanisms underlying the protective effect of exenatide, whereby exenatide reduces S1PR2 expression. Our study identified the novel mechanism of action of exenatide, and the involvement of S1PR2 in HG-induced hRVEC injury. These provide the molecular basis for further investigation of exenatide functions and might show a potential new target for treating DR.
The effects of exenatide on HG-induced hRVECs was investigated through analyzing ROS, inflammatory markers and cell apoptosis in the present study. Our data shows that exenatide reduced the protein levels of inflammationrelated markers IL-1 $\beta$, TNF- $\alpha$ and IL-6, ROS levels and cell apoptosis. These observations were consistent with a previous study implicating exenatide in the regulation of inflammation, apoptosis and oxidative stress. ${ }^{13}$ Reactive oxygen species production is a part of the defense mechanism of the body, with a maintained balance between production and elimination. However, under certain pathological conditions, the antioxidant defense ability of the body decreases, and thus its oxidation capabilities exceed its antioxidant capabilities. Oxidative stress is considered to play a key role in DR development, which could destroy the integrity of the cell membrane, promote cell apoptosis and cause microvascular damage. ${ }^{14,15}$ In DR, hyperglycemia leads to abnormal production of ROS, which further contributes to local inflammation and cell apoptosis. ${ }^{12}$ Furthermore, there is a vicious cycle of positive feedback involving ROS, inflammation and cell apoptosis that is closely correlated with the development of DR. The present study reveals that overproduction of ROS caused by HG induction was decreased by exenatide, and that this repression was counteracted by S1P, an agonist of S1PR2. Moreover, JTE-013, an antagonist of S1PR2, also exhibited an inhibitory effect on ROS production. These observations show the involvement of S1PR2 in exenatidedecreased ROS levels.

The S1PR2 has been reported to regulate phosphorylation of Akt and endothelial nitric oxide synthase (eNOS), ${ }^{5}$ and AKT can also activate eNOS in endothelial cells, ${ }^{16}$ suggesting S1PR2 could regulate Akt-eNOS, as 1 study 


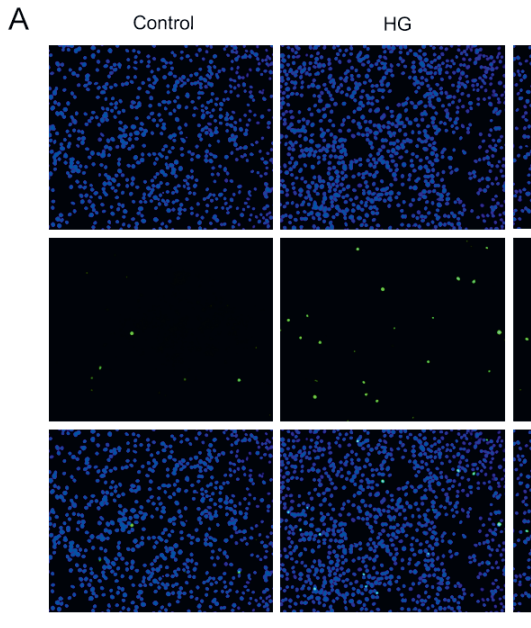

C

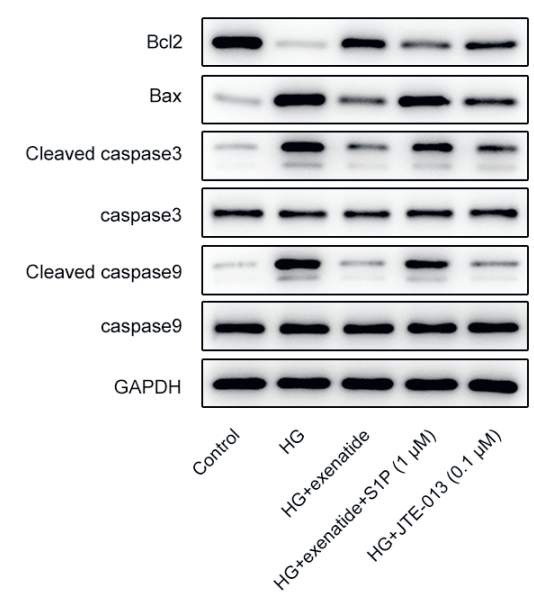

HG+exenatide
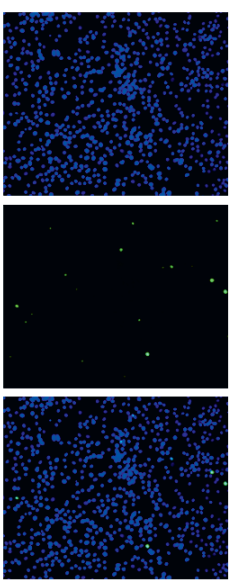

HG+exenatide+S1P(1 $\mu M) \quad H G+J T E-013(0.1 \mu M)$
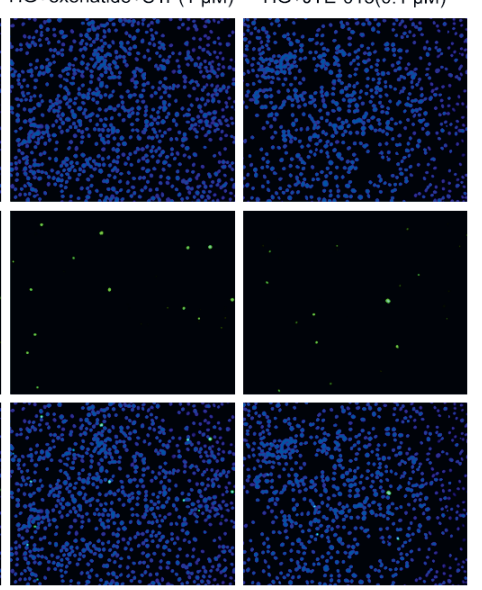

B

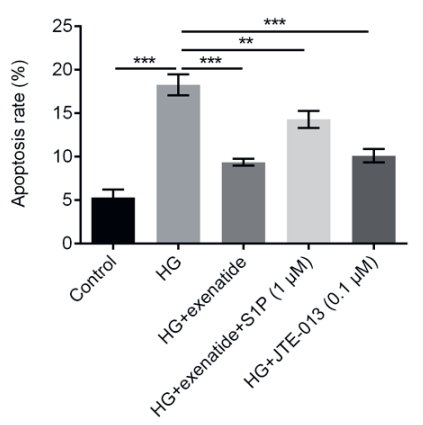

Merged

DAPI

Tunel
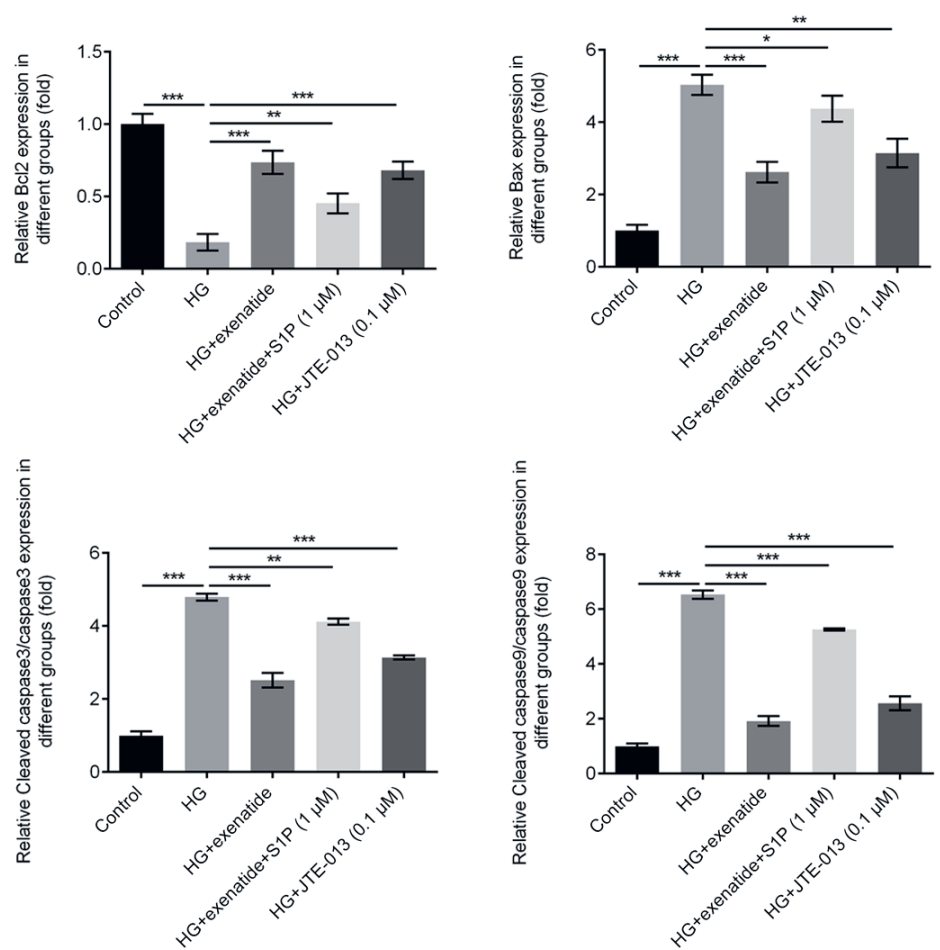

Fig. 4. A and B. TUNEL staining was utilized to analyze cell apoptosis levels; C. Changes in apoptosis-related protein levels (Bcl-2, Bax, cleaved caspase-3, caspase-3, cleaved caspase-9, and caspase-9) after different treatments was visualized using western blot analysis. The results are shown as mean \pm SD percentage of the control level based on 3 independent tests

${ }^{*} p<0.05 ;{ }^{* *} p<0.01 ;{ }^{* * *} p<0.001$.

has indicated. ${ }^{5}$ The modulation of AKT signals is involved in the regulation of ROS and apoptosis in retinal endothelial cells under HG exposure. ${ }^{17}$ Therefore, the effect of exenatide reducing S1PR2 expression, and in turn inhibiting ROS, inflammatory makers and cell apoptosis, might be mediated by the involvement of AKT signals, which requires further study.

A previous study demonstrated protective roles of GLP-1 receptor agonists in preventing retinal neurodegeneration, including neural apoptosis. ${ }^{18}$ The GLP-1 receptor agonists reduce Bax expression and upregulate caspase- 8 levels, while also increasing BCLxl levels. ${ }^{18}$ In addition, exenatide has been reported to protect retinal ganglion cells from HG-induced injury. ${ }^{19}$ Our study demonstrates that exenatide can also regulate $\mathrm{Bcl} 2$, Bax, cleaved caspase-3, and cleaved caspase- 9 levels in hRVECs, which could be blocked by S1P. Similar results were also observed in HGtreated hRVECs after JTE-013 treatment. Therefore, we conclude that exenatide reduced HG-activated apoptosis in hRVECs through suppression of S1PR2 production. The pro-apoptotic role of S1PR2 has been investigated in several studies. ${ }^{20-22}$

\section{Limitations}

The study of the regulatory role of S1PR2 was limited to in vitro hRVECs model of DR; in vivo models of DR still require further study. 


\section{Conclusions}

Presently, the main strategy for DR treatment is to protect retinal microvasculature from injury resulting from diabetes. Our study implies a possible mechanism for exenatide and presents a S1PR2 as a novel molecular target for delaying DR progression.

\section{ORCID iDs}

Yunyun Yang (1) https://orcid.org/0000-0001-5593-0086

Qingyi Zhao (1) https://orcid.org/0000-0002-2303-6546

\section{References}

1. Yamagishi S, Fukami K, Matsui T. Crosstalk between advanced glycation end products (AGEs)-receptor RAGE axis and dipeptidyl peptidase4 -incretin system in diabetic vascular complications. Cardiovasc Diabetol. 2015;14:2, doi:10.1186/s12933-015-0176-5

2. Kahn SE, Haffner SM, Hesse MA, et al. Glycemic durability of rosiglitazone, metformin, or glyburide monotherapy. N Eng J Med. 2006; 355(23):2427-2443. doi:10.1056/NEJMoa066224

3. Huang S, Ma S, Ning M, et al. TGR5 agonist ameliorates insulin resistance in the skeletal muscles and improves glucose homeostasis in diabetic mice. Metabolism. 2019;99:45-56. doi:10.1016/j.metabol. 2019.07.00

4. Yang $Z$, Xiong $F$, Wang $Y$, et al. TGR5 activation suppressed S1P/S1P2 signaling and resisted high glucose-induced fibrosis in glomerular mesangial cells. Pharmacol Res. 2016;111:226-236. doi:10.1016/j. phrs.2016.05.035

5. Cui $\mathrm{H}, \mathrm{Okamoto} Y$, Yoshioka $\mathrm{K}$, et al. Sphingosine-1-phosphate receptor 2 protects against anaphylactic shock through suppression of endothelial nitric oxide synthase in mice. J Allergy Clin Immunol. 2013; 132(5):1205-1214.e1209. doi:10.1016/j.jaci.2013.07.026.

6. Obinata $\mathrm{H}, \mathrm{Hla} \mathrm{T}$. Sphingosine 1-phosphate in coagulation and inflammation. Semin Immunopathol. 2011;34(1):73-91. doi:10.1007/ s00281-011-0287-3

7. Japtok L, Schmitz El, Fayyaz S, Krämer S, Hsu LJ , Kleuser B. Sphingosine 1-phosphate counteracts insulin signaling in pancreatic $\beta$-cells via the sphingosine 1-phosphate receptor subtype 2. FASEB J. 2015; 29(8): 3357-3369. doi:10.1096/fj.14-263194

8. Imasawa T, Koike K, Ishii I, Chun J, Yatomi Y. Blockade of sphingosine 1-phosphate receptor 2 signaling attenuates streptozotocin-induced apoptosis of pancreatic beta-cells. Biochem Biophys Res Commun. 2010;392(2):207-211. doi:10.1016/j.bbrc.2010.01.016

9. Wang W, Lo ACY. Diabetic retinopathy: Pathophysiology and treatments. Int J Mol Sci. 2018;19(6):1816. doi:10.3390/ijms19061816
10. Madsen-Bouterse SA, Kowluru RA. Oxidative stress and diabetic retinopathy: Pathophysiological mechanisms and treatment perspectives. Rev Endocr Metab Disord. 2008;9(4):315-327. doi:10.1007/s11154-0089090-4

11. Osborne NN, Casson RJ, Wood JPM, et al. Retinal ischemia: Mechanisms of damage and potential therapeutic strategies. Prog Retin Eye Res. 004;23(1):91-147. doi:10.1016/j.preteyeres.2003.12.001

12. Wu MY, Yiang GT, Lai TT, Li CJ. The oxidative stress and mitochondrial dysfunction during the pathogenesis of diabetic retinopathy. Oxid Med Cell Longev. 2018;2018:3420187. doi:10.1155/2018/3420187

13. Fang J, Tang $Y$, Cheng $X$, et al. Exenatide alleviates adriamycin-induced heart dysfunction in mice: Modulation of oxidative stress, apoptosis and inflammation. Chem Biol linteract. 2019;304:186-193. doi:10.1016/j.cbi.2019.03.012

14. Madsen-Bouterse SA, Zhong Q, Mohammad G, Ho YS, Kowluru RA. Oxidative damage of mitochondrial DNA in diabetes and its protection by manganese superoxide dismutase. Free Radical Res. 2010;44(3): 313-321. doi:10.3109/10715760903494168

15. Bonnefont-Rousselot D. Glucose and reactive oxygen species. Curr Opin Clin Nutr Metab Care. 2002;5(5):561-568. doi:10.1097/00075197200209000-00016

16. Huang W, Yan Z, Li D, Ma Y, Zhou J, Sui Z. Antioxidant and anti-inflammatory effects of blueberry anthocyanins on high glucose-induced human retinal capillary endothelial cells. OxidMed Cell Longev. 2018; 2018:1862462. doi:10.1155/2018/1862462

17. Xie W, Zhou P, Qu M, et al. Ginsenoside Re attenuates high glucoseinduced RF/6A injury via regulating PI3K/AKT inhibited HIF-1a/VEGF signaling pathway. Front Pharmacol. 2020;11:695. doi:10.3389/fphar. 2020.00695

18. Hernández C, Bogdanov P, Corraliza L, et al. Topical administration of GLP-1 receptor agonists prevents retinal neurodegeneration in experimental diabetes. Diabetes. 2016;65(1):172-187. doi:10.2337/ db15-0443

19. Hao M, Kuang HY, Fu Z, Gao XY, Liu Y, Deng W. Exenatide prevents high-glucose-induced damage of retinal ganglion cells through a mitochondrial mechanism. Neurochem Int. 2012;61(1):1-6. doi:10. 1016/j.neuint.2012.04.009

20. Webster CR, Anwer MS. Hydrophobic bile acid apoptosis is regulated by sphingosine-1-phosphate receptor 2 in rat hepatocytes and human hepatocellular carcinoma cells. Am J Physiol Gastrointest Liver Physiol. 2016;310(10):G865-G873. doi:10.1152/ajpgi.00253.2015

21. Hendley AM, Wang YJ, Polireddy K, et al. p120 catenin suppresses basal epithelial cell extrusion in invasive pancreatic neoplasia. Cancer Res. 2016;76(11):3351-3363. doi:10.1158/0008-5472.can-15-2268

22. Liu K, Cui K, Feng H, et al. JTE-013 supplementation improves erectile dysfunction in rats with streptozotocin-induced type I diabetes through the inhibition of the rho-kinase pathway, fibrosis, and apoptosis. Andrology. 2020;8(2):497-508. doi:10.1111/andr.12716 